\title{
La sindrome anemica-cardio-renale. Seconda parte: diagnostica
}

Giornale di Tecniche Nefrologiche e Dialitiche 2018, Vol. 30(I) 40-46

(C) The Author(s) 2018

Reprints and permissions:

sagepub.co.uk/journalsPermissions.nav

DOI: 10.1 177/0394936218766535

journals.sagepub.com/home/gtn

\section{(SAGE}

\author{
Rodolfo Fernando Rivera', Maria Teresa Sciarrone Alibrandi², \\ Luca Di Lullo ${ }^{3}$, Fulvio Floccari ${ }^{4}$, Antonio De Pascalis ${ }^{5}$, \\ Antonio Bellasi ${ }^{6}$ e Claudio Ronco ${ }^{7}$
}

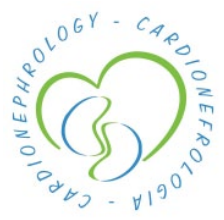

\begin{abstract}
In Part One of this two-part series, the clinical and epidemiological aspects of the cardiorenal anemia syndrome (CRAS) were discussed. Anemia is a complication frequently associated with chronic heart failure (CHF) and chronic kidney disease (CKD).

Part Two of this review focuses on the diagnostic elements of anemia in cardiorenal syndrome (CRS). Bone marrow biopsy remains the gold standard for the assessment of iron ( $\mathrm{Fe})$ stores. However, many other laboratory tests are available, both biochemical and hematological, that are less invasive, more practical and useful for the diagnosis and gradation of iron deficiency (ID). Biochemical tests are based on Fe metabolism and allow the identification of ID before the onset of anemia. Hematologic examinations, on the other hand, based on the morphologic characteristics of the red blood cells are more readily available. New tests are currently available for ID diagnosis before anemia is present. All of these tests are used to determine the type and cause of anemia in a patient with CRS. Unfortunately, there is no single test capable of establishing the diagnosis of ID with or without anemia, but it is necessary to resort to a combination of assessments adapted to the patient's specific clinical situation. Indeed, every assessment aimed at evaluating the iron profile expresses a different aspect of each compartment of the total body Fe (deposit, transport, metabolic-functional, etc.).
\end{abstract}

Using the various available tests improves diagnostic specificity and enhances differential diagnosis.

\section{Keywords}

Cardiorenal anemia syndrome (CRAS), chronic kidney disease (CKD), chronic heart failure (CHF), iron therapy, erythropoiesis-stimulating agents, blood transfusions

\section{Introduzione}

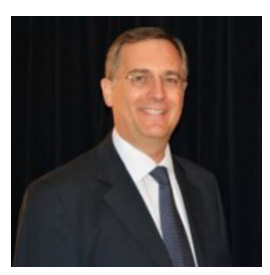

Il capitolo precedente ${ }^{1}$ si è focalizzato sugli aspetti clinici ed epidemiologici della sindrome anemica cardiorenale (CRAS), enfatizzando sulla frequente associazione di questa complicanza clinica nelle malattie croniche come lo scompenso cardiaco congestizio (CHF) e la malattia renale cronica (CKD).

L'anemia può rappresentare una complicanza presente in ognuno dei diversi tipi di sindrome cardiorenale (CRS). ${ }^{1,2}$
IUO Nefrologia e Dialisi, Ospedale San Gerardo Hospital, ASST Monza, Monza

¿UO Nefrologia, Dialisi e Ipertensione, IRCCS Ospedale San Raffaele, Milano

${ }^{3}$ UO Nefrologia e Dialisi, Ospedale L. Parodi Delfino,

Colleferro, Roma

${ }^{4}$ UO Nefrologia e Dialisi, Ospedale San Paolo, Civitavecchia

5 UO Nefrologia e Dialisi, Ospedale V. Fazzi, Lecce

¿UO Nefrologia e Dialisi, Ospedale Sant'Anna, ASST-Lariana, Como

7 O Nefrologia e Dialisi, Ospedale San Bortolo \& International Renal Research Institute Vicenza (IRRIV), Vicenza

\section{Corrispondenza:}

Rodolfo F. Rivera, Divisione di nefrologia dialisi ospedale san Gerardo Hospital, ASST Monza, via Pergolesi, 33, 20900 Monza (MB)

E-mail: rodolfofrivera@gmail.com 
Per tale motivo è necessario impostare un approccio diagnostico adeguato volto a identificare la causa principale dell'anemia e a stabilire la diagnosi differenziale. Nella CRS, l'anemia si sviluppa parallelamente alla progressione della malattia renale e/o cardiaca ed è principalmente causata da una produzione inadeguata di eritropoietina endogena (EPO) o di altri fattori concomitanti come il deficit di ferro (ID), acido folico, vitamina B12, emorragie, infezioni e infiammazione.

La diagnosi di anemia viene posta attraverso una serie di esami di laboratorio che consentono di stabilirne la gravità e la natura. ${ }^{3}$ La diagnosi differenziale non è sempre facile e richiede talvolta uno specifico know-how e sofisticati metodi diagnostici. Il procedimento diagnostico proposto nella presente revisione è articolato su un modello diagnostico fisiopatologico descrittivo, che ha l'obiettivo di definire il principale meccanismo patogenetico dell'anemia. Frequentemente i dati clinicoanamnestici consentono di arrivare rapidamente alla diagnosi, anche senza aver percorso tutto l'iter diagnostico fisiopatologico. ${ }^{4}$

Una volta stabilita la diagnosi dell'anemia nella CRS è possibile impostare una terapia adeguata.

\section{Diagnosi e valutazione clinica dell'anemia nella CRS}

Stabilita la presenza dell'anemia nel paziente affetto da CRS, il test ideale di valutazione dovrebbe tener conto dell'aspetto eziologico e accuratamente differenziare cause associate come l'ID, l'anemia da malattia cronica, le anemie microcitiche e quelli macrocitiche.

Gli esami di laboratorio disponibili per la diagnosi dell'anemia e di ID nei suoi diversi stadi sono molteplici e possono essere didatticamente catalogati come:

- esami ematologici: morfologia del sangue periferico, volume globulare medio (VGM), reticolociti, valutazione dell'emoglobina $(\mathrm{Hb})$ e dell'ematocrito $(\mathrm{Ht})$, morfologia del midollo osseo, indici eritrocitari, indici reticolocitari.

- esami biochimici: sideremia, ferritina, ferritina eritrocitaria e zincoprotoporfirina (ZPP), transferrina, ferritina, recettore solubile della transferrina.

\section{Volume globulare medio (MCV)}

Il volume globulare medio (MCV) rappresenta il primo parametro utile nella flow-chart diagnostica ed è misurato di routine dai contatori automatici. Il MCV consente di distinguere le anemie microcitiche, nelle quali la sintesi $\mathrm{Hb}$ è ridotta per inadeguato apporto di ferro al midollo eritroide o per deficitaria sintesi della globina o dell'eme, da tutte le altre anemie. Il MCV fornito dai contaglobuli è un valore medio, e come tale deve essere valutato nel contesto clinico del paziente. Un valore ridotto di MCV $(<80 \mathrm{fL})$, suggerisce una anemia microcitica. Date le possibile opzioni diagnostiche, per valori tra 80 a $83 \mathrm{fL}$ si consiglia una rivalutazione nel tempo. Valori normali $(80-100 \mathrm{fL})$ o superiori ai $100 \mathrm{fL}$ suggeriscono una anemia normocitica o macrocitica, rispettivamente. ${ }^{5}$

Le anemie microcitiche sono di frequente osservazione, e rappresentano più della metà di tutte le forme di anemie. Il principale eziologico di queste anemie è dato dalla sintesi deficitaria di $\mathrm{Hb}$, dovuta a cause diverse. ${ }^{6} \mathrm{I}$ tre tipi principali di anemia microcitica sono l'anemia sideropenia; l'anemia microinfiammatoria (AMI) o le anemia delle malattie croniche (AMC); e le sindromi talassemiche dovute a deficitaria sintesi di una o più catene globiniche. ${ }^{5} \mathrm{~A}$ questi tipi di anemia microcitica vanno aggiunte altre forme più rare, quali l'anemia saturnina (provocata dall'inibizione della sintesi dell'eme indotta dal piombo), le anemie sideroblastiche congenite (dovute a sintesi deficitaria dell'eme) ed alcune emoglobinopatie, quali l'emoglobina C (dovuta a cristallizzazione dell'emoglobina) e l'emoglobina E (sindrome talassemica a tutti gli effetti).

Le anemie più normo-macrocitiche sono quelle dovute a un disordine primitivo del midollo eritroide, secondarie a malattie sistemiche non ematologiche. Queste processi hanno una patogenesi multifattoriale e sono spesso complicate da perdite ematiche, ID e carenza di follati. Come illustrato nella Tabella 1, esistono diversi tipi di anemie macrocitiche che possono classificarsi secondo il meccanismo fisiopatologico che le produce. L'AMI o AMC sono tra le più comuni di queste anemie secondarie ipoproliferative, ed è causata dall'eccessiva produzione di citochine proinfiammatorie quali interleuchina 1 (IL1), interleuchina 6 (IL3) e fattore di necrosi tumorale (tumor necrosis factor, TNF), di comune riscontro sia nelle malattie infettive croniche che in quelle infiammatorie e/o neoplasie. ${ }^{7}$ Nella CKD l'anemia è causata da un deficit nella produzione di EPO. Le anemia nelle malattie neoplasiche possono essere causate attraverso meccanismi diversi come l'iperproduzione di citochine (AMC), l'infiltrazione diretta del midollo osseo o le perdite ematiche croniche. Altri tipi di anemia ipoproliferativa sono quelle associate alle epatopatie, endocrinopatie, malnutrizione, sindromi mielodisplastiche, mieloproliferative e linfoproliferative e nelle anemie aplastiche.

Esistono altre due forme di anemia macrocitiche: quelle da eritropoiesi inefficace come le anemia megaloblastiche dove la carenza di vitamina B12 o folati sono la caratteristica principale, le sideroblastiche acquisite e le anemia diseritropoietiche congenite. Queste anemie si caratterizzano per una ridotta percentuale di reticolociti $(<3 \%)$ con aumento della bilirubina non coniugata e del LDH. Quando la conta di reticolociti supera il 3\%, in presenza di bilirubina non coniugata e di LDH aumentate, vanno considerate le anemia 
Tabella I. La sindrome anemica cardio renale.

\begin{tabular}{|c|c|}
\hline & EMIA MICROCITICHE (MCV < $80 \mathrm{fL})$ \\
\hline$\checkmark$ & Anemia da carenza marziale \\
\hline$\checkmark$ & Anemia delle malattie croniche \\
\hline$\checkmark$ & Sindromi talassemiche \\
\hline$\checkmark$ & Anemia saturnina \\
\hline$\checkmark$ & Anemia sideroblastica congenita \\
\hline$\checkmark$ & Emoglobina C \\
\hline & Emoglobina $\mathrm{E}$ \\
\hline & $\begin{array}{l}\text { IEMIA NORMOCITICHE (MCV 80-100 fL) e } \\
\text { CROCITICHE (MCV 80-100 fL) }\end{array}$ \\
\hline$\checkmark$ & Anemie ipoproliferative (reticolociti <2\%) \\
\hline & - Anemia delle malattie croniche \\
\hline & - Anemia da insufficienza renale cronica \\
\hline & - Anemia associata ad epatopatia \\
\hline & - Anemia associata ad endocrinopatia \\
\hline & - Anemia associata a malnutrizione \\
\hline & - Anemia refrattarie (sindromi mielodisplosiche) \\
\hline & - Anemia associata a malattia mieloproliferativa \\
\hline & - Anemia associata a malattia linfoproliferative \\
\hline & - Anemia aplastica \\
\hline$\checkmark$ & $\begin{array}{l}\text { Anemie da eritropoiesi inefficace (reticolociti }<3 \% \text {, } \\
\text { bilirrubina non coniugata ed LDL } \uparrow \text { ) }\end{array}$ \\
\hline & - Anemia megaloblastiche (carenza di vit. BI2 o folati) \\
\hline & - Anemia sideroblastiche acquisite \\
\hline & - Anemia diseritropoietiche congenite \\
\hline$\checkmark$ & $\begin{array}{l}\text { Anemie emolitiche (reticolociti }>3 \% \text {, bilirrubina } \\
\text { non coniugata ed LDL } \uparrow \text { ) }\end{array}$ \\
\hline & - Anemia immunoemolitiche \\
\hline & $\begin{array}{l}\text { - Anemia emolitiche da disordine di membrana } \\
\text { eritrocitaria }\end{array}$ \\
\hline & - Anemia emolitiche da emoglobinopatia \\
\hline & - Anemia emolitiche da difetti metabolici eritrocitari \\
\hline & - Emolisi intravascolare \\
\hline$\checkmark$ & Anemia emorragiche \\
\hline
\end{tabular}

emolitiche. Tra le più comuni si segnalano le anemie immunoemofiliche, i disordini della membrana eritrocitaria, le emoglobinopatie, i difetti metabolici eritrocitari e le emolisi intravascolari.

Infine in presenza di reticolociti variabilmente aumentati vanno considerate le anemia emorragiche.

\section{Reticolociti}

Il conteggio dei reticolociti è utile per differenziare fra anemie emolitiche e quelle ipoproliferative o da eritropoiesi inefficace. Nelle prime, il numero di reticolociti appare adeguato per il livello di anemia, lo quale esprime una adeguata risposta del midollo eritroide allo stimolo eritropoietinico (5). Nel secondo caso, il midollo eritroide non è in grado di produrre un numero adeguato di reticolociti. Non esistono criteri universalmente accettati per definire l'adeguatezza della risposta reticolocitaria, ma in generale si considera una risposta adeguata al grado di anemia per valori $>3 \%$. Valori inferiori al $3 \%$ e, soprattutto, inferiori al $2 \%$, suggeriscono una risposta inadeguata. In modo alternativo è possibile considerare il numero assoluto di reticolociti: si considerano adeguati valori $150 \times 109 / \mathrm{L}$, e inadeguati valori inferiori a tele limite.

Target di emoglobina $(\mathrm{Hb})$ ed ematocrito $(\mathrm{Ht})$. La mancanza di una definizione univoca di anemia nella CRS rappresenta un problema di difficile gestione, particolarmente al momento di decidere un trattamento. Generalmente, nell'adulto la diagnosi di anemia si pone quando l' $\mathrm{Hb}$ è inferiore a 13,0 g/dL nel maschio e a $12 \mathrm{~g} / \mathrm{dL}$ nella donna. Il livello di $\mathrm{Hb}$ tende a ridursi progressivamente con l'avanzare dell'età, e nell'anziano $12 \mathrm{~g} / \mathrm{dL}$ costituisce un livello discriminante valido per entrambi i sessi. ${ }^{8-10}$

Per quanto riguarda la $\mathrm{CKD}$, la definizione dei target sembrano essere più chiari. Le linee guida della National Kidney Foundation (NKF) Dialysis Outcomes Quality Initiative (DOQI) per l'anemia inizialmente emesse nel 1997, con successive revisioni nel 2001 e nel $2006,{ }^{8-10}$ e l'aggiornamento del 2007 raccomandava che il target di $\mathrm{Hb}$ doveva essere tra 11 e $12 \mathrm{~g} / \mathrm{dL}$ e mai eccedere i $13 \mathrm{~g} /$ dL. ${ }^{3}$ Le linee guida Kidney Disease Improving Global Outcomes (KDIGO) del $2012^{8}$ raccomandavano che $\mathrm{i}$ pazienti CKD-5D dovessero mantenere concentrazioni di $\mathrm{Hb} \geq 10 \mathrm{~g} / \mathrm{dL}$. In quelli con $\mathrm{Hb}<10 \mathrm{~g} / \mathrm{dL}$, si consigliava l'inizio della terapia in base alla risposta della terapia marziale, al rischio di trasfusione, a quello legato alla terapia con agente stimolante l'eritropoiesi (erythropoiesis-stimulating agent, ESA) e alla presenza di sintomi. Per i pazienti CKD-5 non-D l'inizio della terapia veniva consigliato per concentrazioni di $\mathrm{Hb}<10 \mathrm{~g} / \mathrm{dL}$.

L'anemia è di comune riscontro nei pazienti con CHF, particolarmente in quelli ospedalizzati. È più comune nelle donne, negli anziani e nei pazienti con CKD ed è associato a rimodellamento miocardico avanzato, infiammazione e sovraccarico di volume. ${ }^{11}$ Le recenti linee guida dell' European Society of Cardiology sottolineano la necessità di uno screening per i pazienti con CHF e ID (anemici o non anemici), senza però definire i target di $\mathrm{Hb}$ da raggiungere per ogni stadio di malattia. ${ }^{12}$ Nei pazienti affetti da CHF, la ID si associa a una prognosi peggiore. ${ }^{13}$

Esame del midollo osseo. L'aspirato midollare e/o la biopsia ossea permettono di valutare l'accumulo di ferro nell'interstizio e nei macrofagi midollari e la presenza di sideroblasti.

L'assenza di Fe consente la diagnosi di ID senza la necessità di ricorrere ad altri test di laboratorio. L'esame del midollo osseo è ancora considerato il "gold standard" per la diagnosi di ID, altamente specifico e ampiamente sperimentato, nonostante le sue limitazioni quali il costo, l'invasività, la necessità di una lettura attenta e diligente, e una tecnica di colorazione meticolosa, con possibili errori di campionamento e di interpretazione (artefatti). ${ }^{14}$ In condizioni standardizzate ed eseguito da 
Tabella 2. Sviluppo sequenziale della carenza marziale.

\begin{tabular}{ll}
\hline STADIO della SIDEROPENIA & CONDIZIONE CLINICA \\
\hline $1^{\circ}:$ Prelatente & deplezione dei depositi \\
$2^{\circ}:$ Latente o Subclinico & eritropoiesi ferrocarente \\
$3^{\circ}:$ Manifesto & anemia sideropenica (IDA) \\
\hline
\end{tabular}

operatori esperti, l'esame può fornire un'importante informazione diagnostica, ma nella pratica clinica è poco proponibile al solo scopo di diagnosticare una sideropenia. ${ }^{14}$

Striscio periferico. L'anemia sideropenica cronica è caratterizzata da eritrociti piccoli ed ipocromici. Esistono altre caratteristiche morfologiche delle anemie da ID come la presenza di anisopoichilocitosi, precheratociti e di ellissociti ${ }^{15}$. I precheratociti, sono elementi eritrocitari con uno o più vacuoli distinti, dai margini affilati, aderenti alla membrana cellulare, con pallore centrale conservato; gli ellissociti (pencil cell) sono eritrociti ipocromici ed allungati, con l'asse maggiore 3 volte più lungo dell'asse minore. I precheratociti sono presenti nel $78 \%$ delle anemie da ID e nel $13 \%$ delle anemie da malattia cronica, mentre gli ellissociti sono presenti nel $70 \%$ e nel $13 \%$ rispettivamente delle due forme. Tali dati morfologici non possono essere utilizzati come criteri maggiori per la diagnosi delle anemie microcitiche ma solo come aiuto diagnostico al momento dell'esame morfologico dello striscio di sangue periferico.

Per quanto lo striscio periferico sia affidabile in mani esperte, non ha un rapporto costo beneficio tale da farlo proporre nella pratica routinaria come metodo per la diagnosi della sideropenia.

Valutazione dello stato marziale. Si definisce deficit di ferro (ID) un insufficiente apporto di ferro $(\mathrm{Fe})$ rispetto alle richieste dell'eritrone e degli altri tessuti. ${ }^{5}$ L'ID rappresenta il deficit nutrizionale più comune nel mondo, sia nei paesi sviluppati che in quelli in via di sviluppo ed è la principale causa di anemia (circa 2 miliardi di persone secondo l'Organizzazione Mondiale della Sanità (World Health Organization, WHO). Le conseguenze cliniche si esprimono non solo sul piano ematologico, ma possono coinvolgere il sistema cardiovascolare come conseguenza dell'ipossia, il sistema immunitario, quello endocrino, muscolare e le funzioni neurologiche. ${ }^{1}$

Adeguate riserve di Fe sono essenziali per ottimizzare gli effetti della terapia con gli ESA nei pazienti con CKD. Infatti, la diminuzione dei depositi di Fe o la ridotta disponibilità marziale rappresentano le cause più comuni di resistenza all'ESA.

La ID si sviluppa tipicamente attraverso tre stadi sequenziali corrispondenti a livelli di gravità crescente, di cui l'anemia rappresenta solo lo stadio finale: ${ }^{16}$ (tabella 2 )
Nello stadio più precoce (prelatente) i depositi di Fe si esauriscono progressivamente (bilancio del $\mathrm{Fe}$ negativo), ma dato che l'assorbimento intestinale è ancora sufficiente, non si osservano effetti funzionali. Questa fase può essere caratterizzata da bassi livelli di ferritina sierica.

Il secondo stadio (latente o subclinico) è caratterizzato dal esaurimento completo dei depositi con la conseguente compromissione della sintesi di $\mathrm{Hb}$ e dei processi enzimatici metabolici in grado di portare a delle anomalie nelle funzioni fisiologiche (astenia, anoressia, riduzione della capacità lavorativa, ecc.). In questa fase è tipico osservare bassi livelli di sideremia e di saturazione della transferrina (TSAT), aumento della transferrina, della protoporfirina libera eritrocitaria o zincoprotoporfirina (ZPP) e del recettore solubile della transferrina (sTfR). L'Hb e l'MCV tendono a ridursi ma sono ancora nell'ambito di normalità ed eventualmente possono comparire nel sangue periferico delle emazie ipocromiche.

Nell'ultimo stadio (manifesto), l'apporto di Fe è insufficiente e l'eritrone è incapace a mantenere un'adeguata concentrazione di $\mathrm{Hb}$ che scende sotto i valori normali insieme alla riduzione dell'MCV: tale condizione si manifesta con una deplezione assoluta di Fe in tutti i distretti, che determina una anemia sideropenica ipocromica microcitica, caratterizzata da TSAT $<15 \%$ e ferritina $<12 \mu \mathrm{g} / \mathrm{L}$.

In assenza di anemia l'ID è asintomatico e può rimanere tale anche con una lieve o moderata anemia se questa si è instaurata lentamente.

Sideremia. La concentrazione sierica di Fe è un parametro poco sensibile allo stadio di lieve deficit di Fe. La sideremia si riduce inseguito alla deplezione completa dei depositi di Fe e ancora prima che l'Hb si riduca.

La sideremia è un parametro che subisce una forte influenza da fattori analitici (metodo utilizzato e presenza di emolisi), dalle variazioni circadiane (fino al $100 \%$ nelle 24 ore in soggetti sani e con valori più alti verso sera) e infine risente di una mancanza di specificità. Bassi valori di sideremia possono essere osservati in situazioni quali donazioni o perdite di sangue, gravidanza, infezioni, flogosi acute e croniche, shock, febbre, neoplasie, ischemia del miocardico, ${ }^{16}$ mentre i valori possono aumentare transitoriamente dopo l'ingestione di carne o la terapia orale con ferro. Normalmente la sideremia non viene utilizzata come singola e specifica misura, ma in combinazione con altri parametri di misura dello stato del marziale. Dato che la sua utilità è condizionata dalle variazioni precedentemente esposte e non apportando un valore diagnostico aggiunto a quello della ferritinemia, l'impiego di questo test per l'inquadramento diagnostico dell'ID viene consigliato in associazione alla ferritina ed ovviamente - come viene detto più avanti - alla transferrina.

Ferritina: La concentrazione sierica della ferritina è un marker affidabile e specifico dei depositi di $\mathrm{Fe}$ ampiamente utilizzata nella pratica clinica. ${ }^{17}$ Esiste una stretta 
correlazione tra ferritina e Fe mobilizzabile di deposito, con una dimostrata corrispondenza di $1 \mu \mathrm{g} / \mathrm{L}$ di ferritina per ogni 8-10 mg di $\mathrm{Fe}$ di deposito. ${ }^{18}$ In corso di ID, i livelli sierici di ferritina diminuiscono, diventando così il marker singolo più utile e precoce per la valutazione dello stato marziale.

Nelle situazioni in cui la ferritina si comporta come proteina o reattante della fase acuta infiammatoria e come marker tumorale (infiammazioni, infezioni, neoplasie, epatopatie, ecc.), la sua concentrazione sierica aumenta indipendentemente dai depositi di $\mathrm{Fe}$, perdendo di fatto ogni utilità diagnostica nell'ambito dell'assetto marziale. Inoltre, le concentrazioni di ferritina aumentano nell'alcolismo, nelle trasfusioni, nelle terapie marziali, nell'ipertiroidismo e con i contraccettivi orali, mentre diminuiscono prevalentemente in corso di ID, nel deficit di vitamina $\mathrm{C}$ e nell'ipotiroidismo. In assenza di tali condizioni, valori di normalità sono indicativi di sufficiente quantità di Fe. È generalmente accettato che nei pazienti anemici, una concentrazione di ferritina sierica $<20 \mu \mathrm{g} / \mathrm{L}$ sia diagnostica di assenza di depositi di Fe e quindi di anemia sideropenia.

Nel caso che l'aumento della ferritina sia conseguente a fattori indipendenti dallo stato marziale rende problematica la scelta di un cutoff che escluda uno stato di ID. Il range proposto negli anziani tra $30 \mathrm{a} 75 \mu \mathrm{g} / \mathrm{L}$ è probabilmente dovuto alla maggior prevalenza di malattie infiammatorie in questa coorte di soggetti.

Nella diagnosi di ID nei soggetti anemici, il cutoff di 30 a $40 \mu \mathrm{g} / \mathrm{L}$ fornisce un'ottima efficienza diagnostica ${ }^{19}$ (valore predittivo positivo del $92 \%$ e $98 \%$ rispettivamente), anche senza segni clinici di infezione o infiammazione. Nella diagnosi di ID dei soggetti anziani con anemia, valori $<30 \mu \mathrm{g} / \mathrm{L}$ hanno una sensibilità del $48 \% \mathrm{e}$ una specificità del $100 \%$, mentre valori $<65 \mu \mathrm{g} / \mathrm{L}$ hanno una sensibilità dell' $80 \%$ e una specificità del $98 \%$.

La discrepanza tra disponibilità e richieste di Fe midollare come in quelle situazioni che si possono osservare nei soggetti trattati con ESA o con Fe endovenoso (e.v.) rappresenta un'altra condizione in cui la determinazione sierica della ferritina perde valore diagnostico. Le rapide variazioni dei depositi marziali conseguente al trattamento, provocano variazioni della ferritinemia indipendenti dai depositi. Infatti, il trattamento con ESA pur in associazione a terapia con $\mathrm{Fe}$ e.v., induce una rapida caduta della ferritinemia dal 50 al $70 \%$ rispetto al livello base; in questi soggetti valori di ferritina $<100 \mu \mathrm{g} / \mathrm{L}$ si associano a deficit funzionale di $\mathrm{Fe}$ e ridotta risposta all'ESA. ${ }^{20}$

Il target di ferritina serica suggerito per il trattamento con Fe nei pazienti anemici con CKD-5 D raccomandato dalle NKF-KDOQI Clinical Practice Guidelines è $>200$ $\mathrm{ng} / \mathrm{mL}$, mentre per i pazienti CKD-5 non-D: $>100 \mathrm{ng} / \mathrm{mL}$. I pazienti CKD-5 $\mathrm{D}$ con livelli di ferritina superiori ai 400 $\mathrm{ng} / \mathrm{mL}$ mostrano un dosaggio di ESA più basso del $28 \%$ rispetto a quelli con ferritina inferiore ai $200 \mathrm{ng} / \mathrm{mL}$, suggerendo che i livelli di ferritina più alti sono meglio tollerati e migliorano l'efficienza di ESA. $^{5}$ Valori di ferritinemia superiori ai $500 \mathrm{ng} / \mathrm{mL}$ non sembrano dare beneficio al trattamento con ESA.

Ferritina eritrocitaria o zincoprotoporfirina. La ferritina eritrocitaria o zincoprotoporfirina (ZPP) è stata proposta come un marker di maggior utilità rispetto alla ferritina sierica nel differenziare le anemie sideropeniche da quelle delle AMC o AMI. ${ }^{21}$

Il vero problema di questo marker è dato dall'instabilità del metodo di dosaggio di fronte alle modifiche dinamiche della stato marziale. La determinazione della ZPP è sensibile ad interferenze farmacologiche e componenti plasmatici. Inoltre e ha problemi di specificità in quanto aumenta in situazioni diverse dal deficit di Fe quali intossicazione da piombo, infezioni, infiammazioni, anemia emolitica e difetti acquisiti nella sintesi dell' $\mathrm{Hb}$ come nelle mielodisplasie. Queste caratteristiche rendono il parametro poco utilizzabile nella pratica clinica.

Tuttavia, la scarsa variabilità circadiana rispetto a quella osservata per la sideremia e per la TSAT, la semplicità nella misurazione (minima esperienza tecnica e scarsa quantità di sangue, bastano due gocce), rendono questo marker di particolare interesse nelle indagini su popolazioni.

Transferrina e la capacità totale legante il Fe. La transferrina è la proteina che trasporta il Fe. È possibile determinarla mediante metodo immunologico o esprimerla come la capacità totale legante il $\mathrm{Fe}$ (TIBC), ossia, la quantità di Fe aggiunto che può essere legato dal plasma (sideremia moltiplicato per 100 e diviso per la capacità totale di ferro $=$ TIBC). Normalmente, entrambi i valori possono risentire della presenza di alcune situazioni patologiche. ${ }^{22} \mathrm{La}$ sintesi della transferrina è regolata dallo stato marziale e quindi aumenta linearmente fino approssimativamente a valori di $400 \mu \mathrm{g} / \mathrm{L}$ nelle situazioni di deplezione dei depositi ed è ridotta quando i depositi marziali ed è viceversa ridotta quando tali depositi sono aumentati.

Anche la concentrazione della transferrina può essere condizionata da fattori non correlati allo stato marziale come nelle infiammazioni, nelle infezioni, nelle epatopatie, nelle neoplasie, nella sindrome nefrosica e nella malnutrizione. ${ }^{23}$ In queste condizioni, i valori di transferrina si riduce mentre invece aumenta ad esempio in corso di terapia con contraccettivi orali. È questo quindi un parametro specifico ma poco sensibile.

Saturazione transferrinica. Nella pratica clinica, i due test più utilizzati sono la ferritina serica e la saturazione transferrinica (TSAT) ${ }^{24}$. La TSAT\% riflette la percentuale dei siti di transferrina legati dal Fe rispetto a quelli totali; rappresenta pertanto il rapporto tra la sideremia e la TIBC espresso in percentuale.

La molecola di transferrina ( $\mathrm{Tf}$ ) contiene due siti di legame per il trasporto del Fe da siti di deposito a cellule progenitrici della serie rossi. Un valore TSAT del 50\% indica che la metà dei siti di legame sono attivati da Fe. $\mathrm{La}$ 
distinzione che può fornirci il dato di una carenza assoluta e funzionale di Fe nei pazienti con IRC trattati con ESA è essenziale e la valutazione della TSAT\% e la concentrazione di ferritina serica sono assai importanti. Nei soggetti normali, la carenza di Fe è considerata 22assoluta" quando i depositi di ferro sono esauriti (livelli circolanti di ferritina $<12 \mathrm{ng} / \mathrm{mL}$, e la delivery di Fe è compromessa, come indicato da una TSAT inferiore al 16\%). ${ }^{22}$ Nei pazienti con IRC una carenza di Fe viene definita come i valori circolanti di ferritina $<100 \mathrm{ng} / \mathrm{mL}$ e livelli di TSAT inferiori al $20 \%$.

In assenza dei i valori della TIBC, è comunque possibile stimare la quantità di Fe legato in condizione di saturazione della transferrina, considerando che $1 \mathrm{mg}$ di transferrina trasporta al massimo $1.41 \mathrm{~g}$ di ferro. Pertanto:

$$
\begin{gathered}
\mathrm{TSAT}=\mathrm{Fe}(\mu \mathrm{g} / \mathrm{dL}) /(\mathrm{Tf}(\mathrm{mg} / \mathrm{dL}) \times 1.41) \times 100 \text { oppure } \\
\mathrm{TSAT}=\text { Ferro }(\mu \mathrm{g} / \mathrm{dL}) / \mathrm{Tf}(\mathrm{mg} / \mathrm{dL}) \times 70.9
\end{gathered}
$$

La TSAT ha gli stessi limiti della sideremia e della transferrinemia (variazioni diurne e bassa specificità). I valori possono essere influenzati in corso di malattie infiammatorie e quindi non permette di discriminare tra deficit di $\mathrm{Fe}$ e anemia da malattia cronica. Pertanto valori normali o aumentati sono più utili nell'escludere la sideropenia rispetto a di valori bassi nell'identificarli. ${ }^{16}$

Recettore solubile della transferrina (sTfR). La concentrazione sierica del sTfR è proporzionale alla quantità totale di TfR presente a livello cellulare. ${ }^{25}$ Circa 1' $80 \%$ della concentrazione totale di sTfR è presente sulla membrana dei precursori eritroidi, pertanto il valore circolante esprime principalmente l'attività eritroide: si riduce nei casi di ipoplasia eritroide (anemia aplastica, chemioterapia, insufficienza renale) mentre aumenta nell'iperplasia (anemie emolitiche, talassemie). Un valore inappropriatamente basso di sTfR rispetto al grado di anemia è indicativo di riduzione dell'attività eritropoietinica midollare. Nella carenza di Fe la sintesi intracellulare e i livelli sierici del sTfR aumentano sensibilmente.

La ferritina sierica e il sTfR possono ricoprire l'intero spettro diagnostico della carenza marziale: la prima come il marker più sensibile e specifico della riduzione dei depositi e il secondo come il marker più sensibile di eritropoiesi ferro carente. I livelli di sTfR presentano maggior stabilità nella diagnosi di ID funzionali, anche con depositi marziali e concentrazione di ferritina normale, rispetto alla TSAT, ed è più precoce rispetto alla ZPP.

In corso di terapia con ESA, le concentrazioni sieriche di sTfR aumentano in tempi più brevi rispetto all'aumento dell'Hb. ${ }^{26}$ Per tale motivo, alcuni autori hanno attribuito un valore predittivo al sTfR, durante il monitoraggio della terapia con ESA nei pazienti anemici in dialisi.

Il principale vantaggio del sTfR è dato dalla stabilità del marcatore, cioè la sua concentrazione non viene influenzata da processi infiammatori, infettivi o danni epatici e non varia con l'età, il sesso e lo stato di gravidanza; risultando particolarmente utile nel discriminare tra pazienti con ID da forme di anemia delle malattie croniche.

Epcidina. La epcidina è un ormone peptidico di recentemente scoperta considerata come il regolatore chiave dell'ingresso del ferro nel plasma, ${ }^{27}$ è up-regolato da infiammazione e aumentato dalle riserve di ferro e downregolato dalla ID. L'epcidina blocca il rilascio del Fe dai macrofagi, limitando la disponibilità di Fe all'eritropoiesi. Elevati livelli serici delle isoforma epcidina bioattiva sono stati costantemente riportati in pazienti CKD-5 D 28,29 tuttavia, la determinazione dei livelli circolanti di epcidina non ha dimostrato essere clinicamente utile o superiore rispetto agli esami standard per la determinazione dello stato marziale in pazienti con CKD. ${ }^{30}$

\section{Conclusioni}

Per quanto la causa di un'anemia possa emergere dall' anamnesi e dall'esame obiettivo del paziente, il supporto del laboratorio è determinante. Gli esami di laboratorio proposti nella presente revisione consentono di stabilire la diagnosi, la causa, la gravità e la natura dell'anemia nei pazienti affetto da CRS.

Tuttavia, bisogna tener presente che non esiste un singolo esame in grado di stabilire la diagnosi di ID con o senza anemia, ma è necessario ricorrere ad una combinazione di accertamenti adattati alla specifica situazione clinica del paziente. Infatti, ogni accertamento volto a valutare il profilo marziale esprime un diverso aspetto dei compartimenti del Fe corporeo (deposito, trasporto, metabolico-funzionale, ecc.).

L'impiego dei diversi test disponibili migliora la specificità diagnostica, favorisce la diagnosi differenziale e offre uno strumento di grande utilità clinica nel corso della terapia con supplementazione di Fe.

\section{Dichiarazione di assenza di conflitto di interessi}

Gli Autori dichiarano di non avere conflitti di interessi.

\section{Finanziamenti}

Gli Autori dichiarano di non aver ricevuto finanziamenti specifici da qualsiasi ente nei settori pubblico, privato o senza fini di lucro.

\section{Bibliografia}

1. Rivera RF, Sciarrone Alibrandi MT, Di Lullo L, et al. The cardiorenal anemia syndrome. Part one: epidemiology and clinical aspects. G Tec Nefrol Dial 2017; 29: 157-232.

2. Ronco C, McCullough P, Anker SD, et al. Cardio-renal syndromes: report from the consensus conference of the acute dialysis quality initiative. Eur Heart J 2010; 31: 703-711.

3. KDOQI Clinical Practice Guideline and Clinical Practice Recommendations for anemia in chronic kidney disease: 
2007 update of hemoglobin target. Am J Kidney Dis 2007; 50: 471-530.

4. Chapter 1: Diagnosis and evaluation of anemia in CKD. Kidney Int Suppl 2012; 2: 288-291.

5. Rivera RF, Alibrandi MTS, Di Lullo L, et al. Clinical management of anemia in patients with CKD. G Ital Nefrol 2017; 34 (Suppl 69): 20-35.

6. Rivera RF, Di Lullo L, De Pascalis A, et al. Anemia in Patients with Chronic Kidney Disease: Current Screening and management Approaches. Nephrol Ren Dis 2016; 1(1).

7. Weiss G e Goodnough LT. Anemia of chronic disease. $N$ Engl J Med 2005; 352: 1011-1023.

8. National Kidney Foundation. KDOQI Clinical Practice Guideline and Clinical Practice Recommendations for anemia in chronic kidney disease. Am J Kidney Dis 2006; 47 (5 Suppl 3): S1-S146.

9. IV. NKF-K/DOQI Clinical Practice Guidelines for Anemia of Chronic Kidney Disease: update 2000. Am J Kidney Dis 2001; 37 (1 Suppl 1): S182-S238.

10. NKF-DOQI clinical practice guidelines for the treatment of anemia of chronic renal failure. Am J Kidney Dis 1997; 30 (4 Suppl 3): S192-S240.

11. O'Meara E, Rouleau JL, White M, et al. Heart failure with anemia: novel findings on the roles of renal disease, interleukins, and specific left ventricular remodeling processes. Circ Heart Fail 2014; 7: 773-781.

12. Ponikowski P, Voors AA, Anker SD, et al. 2016 ESC Guidelines for the diagnosis and treatment of acute and chronic heart failure: The Task Force for the diagnosis and treatment of acute and chronic heart failure of the European Society of Cardiology (ESC). Eur J Heart Fail 2016; 18: 891-975.

13. Jankowska EA, Kasztura M, Sokolski M, et al. Iron deficiency defined as depleted iron stores accompanied by unmet cellular iron requirements identifies patients at the highest risk of death after an episode of acute heart failure. Eur Heart J 2014; 35: 2468-2476.

14. Barron BA, Hoyer JD e Tefferi A. A bone marrow report of absent stainable iron is not diagnostic of iron deficiency. Ann Hematol 2001; 80: 166-169.

15. Harrington AM, Ward PCJ e Kroft SH. Iron deficiency anemia, beta-thalassemia minor, and anemia of chronic disease: a morphologic reappraisal. Am J Clin Pathol 2008; 129: 466- 471.

16. Cook JD e Skikne BS. Iron deficiency: definition and diagnosis. J Intern Med 1989; 226: 349-355.

17. Lipschitz DA, Cook JD e Finch CA. A clinical evaluation of serum ferritin as an index of iron stores. N Engl J Med 1974; 30; 290: 1213-1216.
18. Jacob RA, Sandstead HH, Klevay LM, et al. Utility of serum ferritin as a measure of iron deficiency in normal males undergoing repetitive phlebotomy. Blood 1980; 56: 786-791.

19. Mast AE, Blinder MA, Gronowski AM, et al. Clinical utility of the soluble transferrin receptor and comparison with serum ferritin in several populations. Clin Chem 1998; 44: 45-51.

20. Brugnara C, Colella GM, Cremins J, et al. Effects of subcutaneous recombinant human erythropoietin in normal subjects: development of decreased reticulocyte hemoglobin content and iron-deficient erythropoiesis. J Lab Clin Med 1994; 123: 660-667.

21. Braun J. Erythrocyte zinc protoporphyrin. Kidney Int Suppl 1999; 69: S57-S60.

22. Gambino R, Desvarieux E, Orth $\mathrm{M}$, et al. The relation between chemically measured total iron-binding capacity concentrations and immunologically measured transferrin concentrations in human serum. Clin Chem 1997; 43: 2408-2412.

23. Ito $\mathrm{S}$ e Ishida Y. [Iron, total iron binding capacity (TIBC), unsaturated iron binding capacity (UIBC)]. Article in Japanese. Nihon Rinsho Jpn J Clin Med 2004; 62 Suppl 12: 288-291.

24. Elsayed ME, Sharif MU e Stack AG. Transferrin Saturation: A Body Iron Biomarker. Adv Clin Chem 2016; 75: 71-97.

25. Pfeiffer CM e Looker AC. Laboratory methodologies for indicators of iron status: strengths, limitations, and analytical challenges. Am J Clin Nutr 2017; 106 (Suppl 6): 1606S1614S.

26. Gaweda AE. Markers of iron status in chronic kidney disease. Hemodial Int Int Symp Home Hemodial 2017; 21 Suppl 1: S21-S27.

27. Nicolas G, Viatte L, Bennoun M, et ak. Hepcidin, a new iron regulatory peptide. Blood Cells Mol Dis 2002; 29: 327-335.

28. Zaritsky J, Young B, Gales B, et al. Reduction of Serum Hepcidin by Hemodialysis in Pediatric and Adult Patients. Clin J Am Soc Nephrol 2010; 5: 1010-1014.

29. Kato A, Tsuji T, Luo J, et al. Association of prohepcidin and hepcidin-25 with erythropoietin response and ferritin in hemodialysis patients. Am J Nephrol 2008; 28: 115-121.

30. Tessitore N, Girelli D, Campostrini N, et al. Hepcidin is not useful as a biomarker for iron needs in haemodialysis patients on maintenance erythropoiesis-stimulating agents. Nephrol Dial Transplant 2010; 25: 3996-4002. 